Asociación de Jóvenes Historiadores y Arqueólogos de Murcia

PANTR REL

REVISTA DE CIENCIA

$$
\begin{array}{r}
Y \\
\text { DIDÁCTICA } \\
\text { DE LA HISTORIA } \\
\text { III - } 2^{2} \text { época }
\end{array}
$$




\section{ASOCIACIÓN DE JÓVENES HISTORIADORES Y ARQUEÓLOGOS DE MURCIA}

\section{PANTA REI. REVISTA DE CIENCIA Y DIDÁCTICA \\ DE LA HISTORIA III. $2^{a}$ época}


CONSEJO EDITORIAL: LAURA ARIAS FERRER

ALEJANDRO EGEA VIVANCOS

ANTONINO GONZÁLEZ BLANCO

RAFAEL GONZÁLEZ FERNÁNDEZ

JOAQUÍN LOMBA MAURANDI

ENRIQUE QUINTANA CIFUENTES

GONZALO MATILLA SÉIQUER

JOSÉ ANTONIO MOLINA GÓMEZ

REDACTOR JEFE:

ÁNGEL LUIS GONZÁLEZ TORRES

Depósito legal: MU-966-1995

I.S.S.N. 1136-2464

Edición de Compobell, S.L. Murcia 


\section{ÍNDICE}

AGRADECIMIENTOS

Presentación

Por Alejandro Egea Vivancos, Laura Arias Ferrer

\section{ARTÍCULOS}

Análisis microscópico de la industria lítica: la traceología

Por Ignacio Martín Lerma

Reflexiones en torno al estudio de la economía en Prehistoria

Por Valentín Martínez García

Aplicaciones SIG en el análisis de las sociedades del pasado. Un caso de estudio: Las primeras comunidades campesinas del Levante Peninsular

Por Gabriel García Atiénzar

La situación actual de los estudios de egiptología en España

Por José Javier Martínez García

La vida cotidiana en el Imperio Asirio. Usos y costumbres de un pueblo que conquistó las Cuatro Regiones del Mundo

Por Ángel Luis González Torres

Las formas de intercambio y las estructuras comerciales orientalizantes en la Vega Baja del Segura: dos variables de estudio arqueológico

Por Sara Pernas García 
Evolución de los patrones de asentamiento en época ibérica. Una propuesta de estudio del mundo ibérico murciano a través del análisis del poblamiento

Hispania Tierra de Roma. Organización y gestión del suelo

Por $M^{a}$ Carmen Santapau Pastor

Hacia una arqueología de la España bizantina. Breves notas a propósito del seminario Work in progress

Por Jaime Vizcaíno Sánchez

Reflexiones acerca de un estudio sobre las estancias auxiliares en la arquitectura barroca catedralicia

Por Francisca del Baño Martínez

La investigación sobre fisiognomía y expresión de las pasiones. Objetivos y metodología

Por María del Mar Albero Muñoz 


\title{
LA VIDA COTIDIANA EN EL IMPERIO ASIRIO Usos y costumbres de un pueblo que conquistó las Cuatro Regiones del Mundo
}

\author{
ÁNGEL LUIS GONZÁLEZ TORRES \\ IPOA - MURCIA
}

\section{Resumen}

El presente trabajo pone de manifiesto la sesión del seminario Work in Progress llevado a cabo en la Facultad de Letras de la Universidad de Murcia dedicada a la investigación historiográfica acerca de la vida cotidiana durante el Imperio Nuevo Asirio, iniciándose con un estudio bibliométrico. Se analiza aquí la metodología empleada en dicha investigación, partiendo de lo general y avanzando en mayores niveles de concreción temática, centrándose posteriormente en uno de sus aspectos, la situación de la mujer en el Imperio Neoasirio. Culmina con algunas de las más destacadas conclusiones obtenidas en dicha investigación.

Palabras clave: Historiografía, Vida cotidiana, Imperio Neoasirio, Bibliometría, Situación de la mujer, Metodología

\begin{abstract}
This paper is a brief summary of the seminar Work in Progress carried out in the University of Murcia regarding the historigraphy of the daily life during the Neoassyrian Empire. The methodology is analysed here, considering a bibliometric study as starting point and moving towards further levels of detail, focussing in one issue at the end: the situation of the Assyrian Women during the Neoassyrian Empire. Finally the paper includes some of the most outstanding conclusions of this investigation.
\end{abstract}

Key words: Historiography, Daily life, Neoassyrian Empire, Bibliometry, Woman's situation, Methodology. 


\section{Introducción}

En mayo de 2007 dentro del seminario Work in Progress afronté la tarea de transmitir a un grupo de compañeros, doctorandos mayoritariamente, las líneas generales de mi investigación acerca de la vida cotidiana en el Imperio Neoasirio.

Siempre resulta una labor difícil introducir a otras personas en algo tan personal como una investigación doctoral, pero el mismo hecho de intentarlo reporta significativas ventajas, haciendo una interesante labor de introspección, algo necesario y que siempre vamos dejando pendiente puesto que otras tareas reclaman nuestra inmediata atención.

Pararse a reflexionar momentáneamente sobre lo que llevamos hecho antes de continuar con ello es un ejercicio que requiere un notable esfuerzo y que, al menos en este caso, recompensa con una mejor visión de conjunto, una mayor claridad de las metas que nos hemos propuesto y, no menos importante, de lo que nos ha motivado a seguir ese camino.

Lo primero que fue analizado para la sesión del seminario fue lo que había motivado a inclinarme por el tema que me ocupaba: la Historia del Próximo Oriente Antiguo, concretamente la del Imperio Asirio Nuevo. Desde que empecé mis estudios de Historia tuve claro mi vocación por la Antigüedad en general y por la Orientalística muy en particular. La fascinación que este tema ha producido sobre mí me llevó a interesarme sobre él desde el principio, parte de la cual debo agradecer a los profesores de esta área de la Universidad de Murcia y a la larga tradición y trayectoria que estos estudios tienen en ella.

Por otro lado, a lo largo los años de carrera desarrollé notablemente el interés por aquellos temas que comúnmente se engloban en ese epígrafe algo difuso al que llamamos vida cotidiana, y que suele hacer referencia a los aspectos que tanto marcan a los ciudadanos comunes en su quehacer diario y que tanto cuesta a veces rastrear en las fuentes históricas, dedicadas en un alto porcentaje a los grandes nombres. Es aquello a lo que Don Miguel de Unamuno llamó intrahistoria, lo que no publican los periódicos pero que tan importante y determinante resulta a menudo.

Hecho esto, mi siguiente paso fue el de ilustrar a los compañeros con los instrumentos que me habían sido necesarios para esta investigación, repasando brevemente aquellos más destacados como el aprendizaje filológico, muy en especial el acadio, lengua franca del Próximo Oriente Antiguo y del Imperio Asirio Nuevo.

Una vez presentados la motivación y los instrumentos de la investigación, sólo queda exponer la misma a los compañeros del seminario, y que culminó con la tesina titulada La vida cotidiana del Imperio Neoasirio en la Historiografía moderna ${ }^{1}$.

1 Tesina dirigida por los Doctores D. Rafael González Fernández y D. Gonzalo Matilla Séiquer, presentada en abril de 2007. 


\section{La historiografía}

El diccionario de la Real Academia ${ }^{2}$ define el término Historiografía con tres fórmulas distintas:

1. Arte de escribir la historia.

2. Estudio bibliográfico y crítico de los escritos sobre historia y sus fuentes, y de los autores que han tratado de estas materias.

3. Conjunto de obras o estudios de carácter histórico.

No cabe duda de que de las tres definiciones es la primera la más hermosa, pues escribir Historia es, o debe ser, un arte, con el que además estimular nuestro conocimiento se estimule también nuestro deleite. Pero es especialmente con la segunda de las definiciones con la que desarrollaremos el grueso de este artículo.

Toda investigación histórica debe empezar con un estudio de lo que otros investigadores, historiadores, arqueólogos o antropólogos hayan publicado sobre el mismo tiempo. De esta forma no sólo se evitan reiteraciones, sino que permite también comenzar la tarea con una mayor base y un conocimiento previo que si bien no debe condicionar, sí debe servir de guía, anticipándonos en ocasiones algunas de las problemáticas con las que nos vamos a encontrar. Es por tanto una labor ineludible y fundamental.

Con la intención de cumplimentar tal labor, comercia esta investigación con una recogida de bibliografía acerca de la vida cotidiana en el Imperio Asirio Nuevo. Para ello partimos de lo más general a lo más concreto: buscando bibliografía en los libros y manuales de los que se disponía y tenía acceso, analizando aquellos que pudiesen estar relacionados en mayor o menor medida con mi objeto de estudio.

Una vez agotada esa vía, hay tres pilares que resultaron fundamentales para la recopilación bibliográfica: en primer lugar la revista Orientalia, cuyo lugar en este tipo de estudios es indiscutiblemente fundamental, y que aporta todo el material bibliográfico publicado sobre el Próximo Oriente Antiguo ordenado por autores y por temas en su sección llamada Keilschriftbibliographie.

En segundo lugar destaca el trabajo llevado a cabo por la Universidad de Helsinki a través del Institute for Asian \& African Studies con la publicación de los Archivos Estatales Asirios (SAA) y las monografías referidas a éstos, destacando su boletín y su base de datos.

Por último hay que resaltar el papel jugado por las Tecnologías de la Información y la Comunicación, con las bases de datos bibliográficas y documentales, entre la que

2 R.A.E. Diccionario de la lengua española. Vigésima segunda edición. 
destaca REBIUN, el Catálogo Colectivo de Red de Bibliotecas Universitarias Españolas.

Partiendo de estos instrumentos, queda elaborado un repertorio bibliográfico de 364 obras de distinta naturaleza, origen y objetivo, que para su correcto análisis y trabajo se organiza en un listado siguiendo una pauta cronológica basada en su año de publicación.

Pero un estudio historiográfico no debe quedarse en un mero listado bibliográfico, sino que debe profundizar, como sostiene la definición.

El primero de los trabajos que se desarrollaron con ese repertorio bibliográfico fue el de dotar al estudio historiográfico del mayor carácter cuantitativo que fuera posible. Para ello se pasó a elaborar un estudio bibliométrico que aportara información estadística y fácilmente cuantificable acerca de tema de estudio, la vida cotidiana durante el Imperio Asirio Nuevo.

\section{Estudio bibliométrico}

Pasamos así a concebir el repertorio bibliográfico como una muestra aleatoria de los trabajos, estudios e investigaciones que acerca de este tema se habían publicado hasta el año 2005, desde los orígenes de la Asiriología como disciplina histórica. La exhaustividad empleada en la búsqueda de la bibliografía dota de la adecuada aleatoriedad a la muestra estadística resultante, lo que permitirá emprender la tarea bibliométrica con ciertas garantías de resultados significativos.

El primer criterio que se empleó para el análisis estadístico fue el cronológico, dado que un primer estudio superficial así lo aconsejaba, por lo que para estructurar el trabajo posterior se compartimentaron la muestra de obras, agrupándolas en las siguientes secciones temporales:

- Hasta 1899

- De 1900 a 1939

- De 1940 a 1969

- De 1970 a 1989

- De 1990 a 2005 


\section{Publicaciones por años}

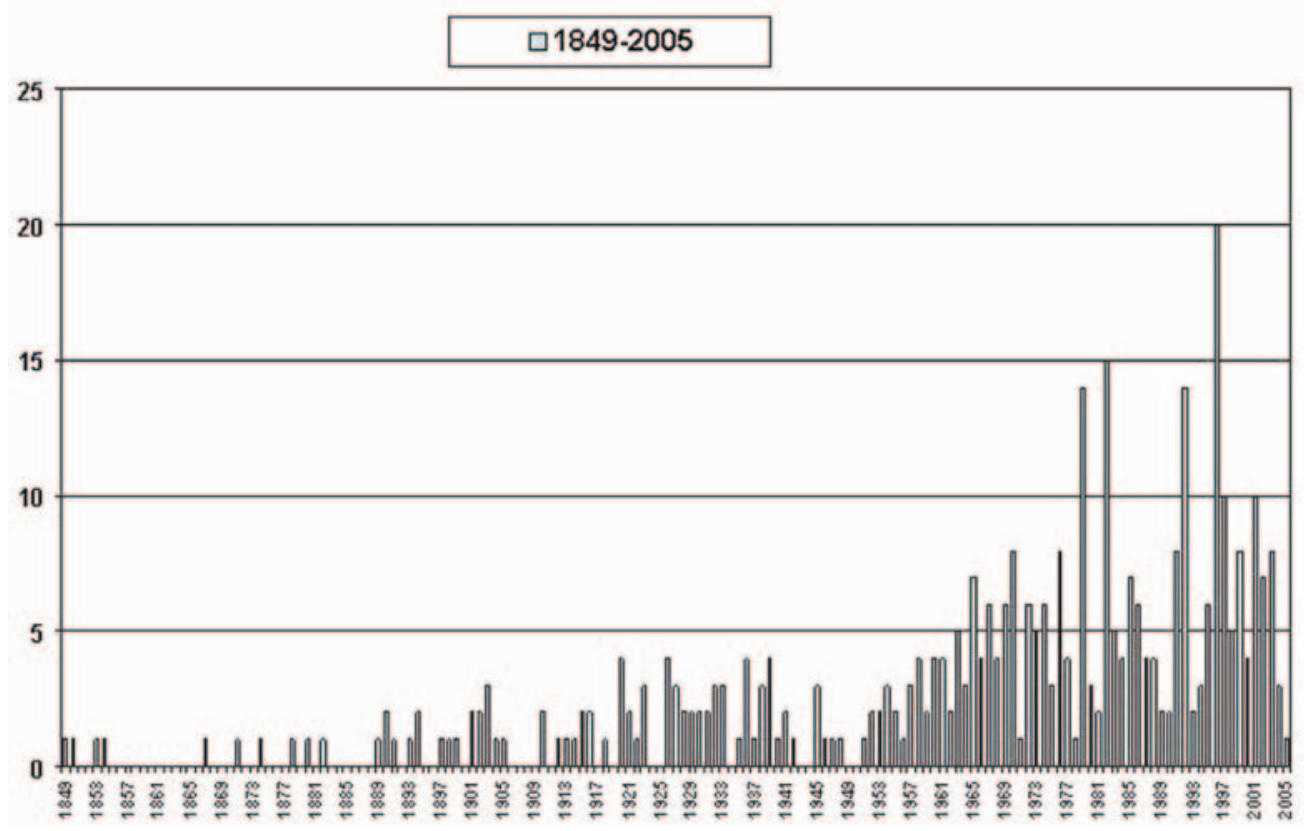

Hecho esto, y tras el anteriormente citado estudio previo, se establecieron los criterios a emplear en el análisis bibliométrico, concretando las siguientes categorías de estudio que servirían posteriormente para estipular pautas o características comunes que nos permitan establecer algunas conclusiones.

Las categorías para el estudio que se emplearon en la investigación fueron las siguientes:

$\rightarrow$ Año de publicación: de esta manera se reincide con mayor profundidad en el criterio cronológico anteriormente citado.

$\rightarrow$ Tipología de la publicación: se establece tres categorías generales, monografía, artículo y manual. 


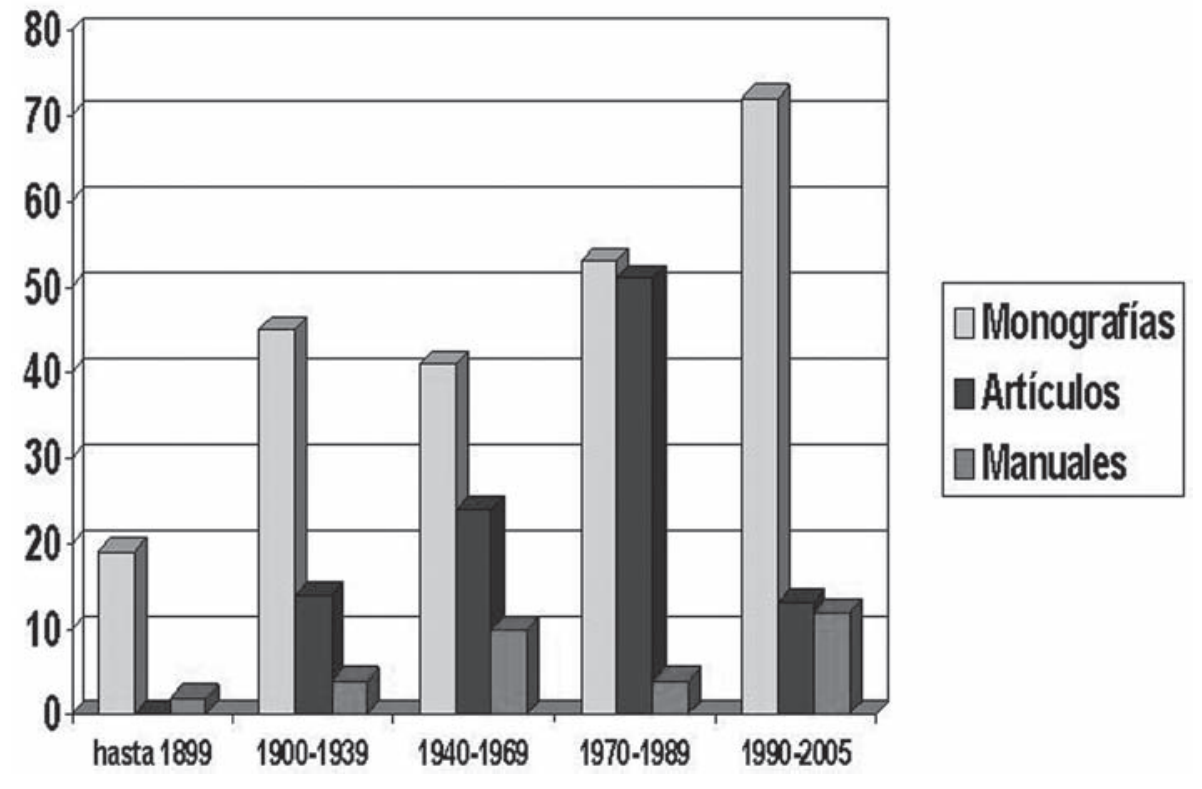

$\rightarrow$ País de publicación original: un aspecto relevante en extremo para conocer la evolución historiográfica de la Asiriología.

\section{Publicaciones por países}

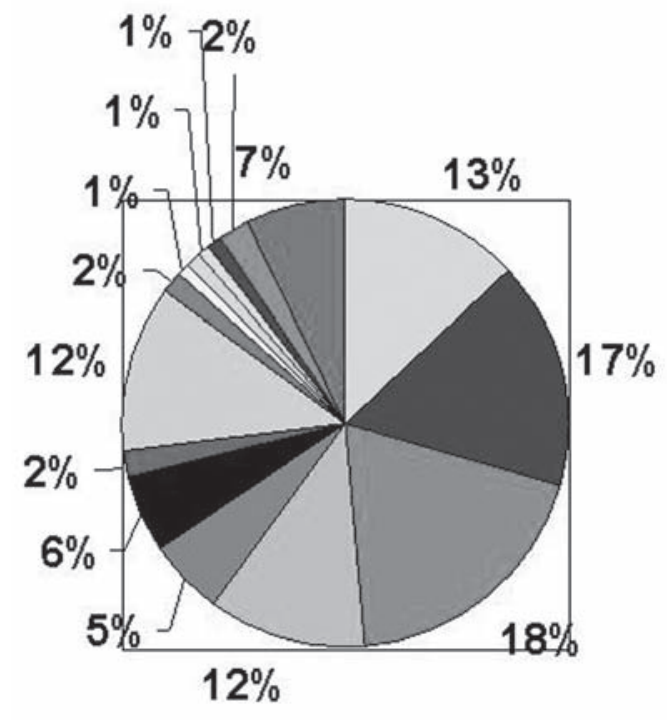

\begin{tabular}{|l|}
\hline$\square$ Reino Unido \\
$\square$ Francia \\
$\square$ Alemania \\
$\square$ EE.UU. \\
$\square$ Países Bajos \\
$\square$ Italia \\
$\square$ Austria \\
$\square$ Finlandia \\
$\square$ Bélgica \\
$\square$ Turquía \\
$\square$ Dinamarca \\
$\square$ Canada \\
$\square$ Suiza \\
$\square$ Otros \\
$\square$ ESPAÑA \\
\hline
\end{tabular}


$\rightarrow$ Idioma original: un aspecto que contrastado con la anterior categoría ofrece una valiosísima información historiográfica.

\section{Publicaciones por idiomas}

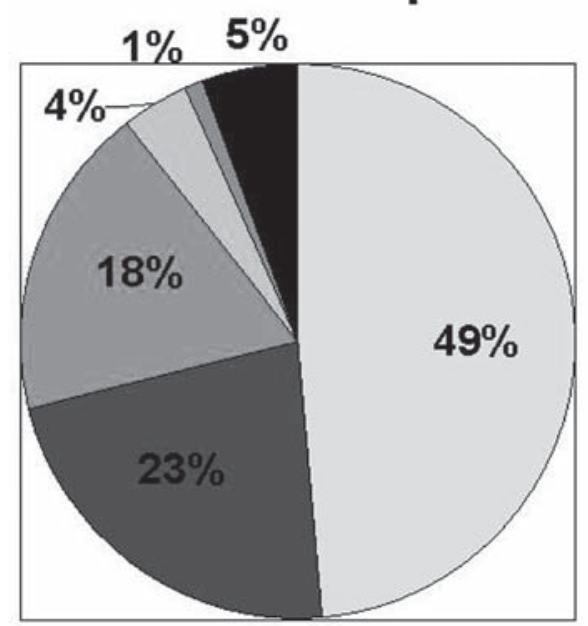

\begin{tabular}{|l|}
\hline Inglés \\
$\square$ Alemán \\
$\square$ Francés \\
$\square$ Italiano \\
$\square$ Otros \\
$\square$ Español \\
\hline
\end{tabular}

A estas cuatro categorías generales se le añadió una más, referente a la situación en España, dentro de la cual hay que destacar dos categorías de estudio con carácter específico por razones de obvia proximidad:

$\rightarrow$ Producción nacional: obras escritas por autores nacionales o editadas por organismos nacionales, concretadas por ciudades.

\section{Publicaciones españolas por ciudad}

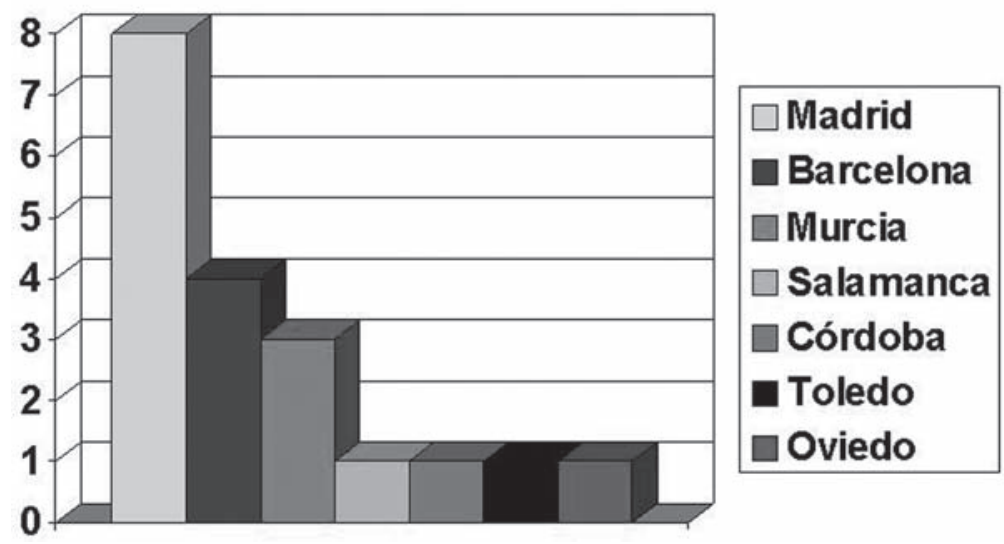


$\rightarrow$ Traducciones: obras escritas en otros idiomas que han sido traducidas y publicadas en castellano.

\section{Idioma original de traducciones al castellano}
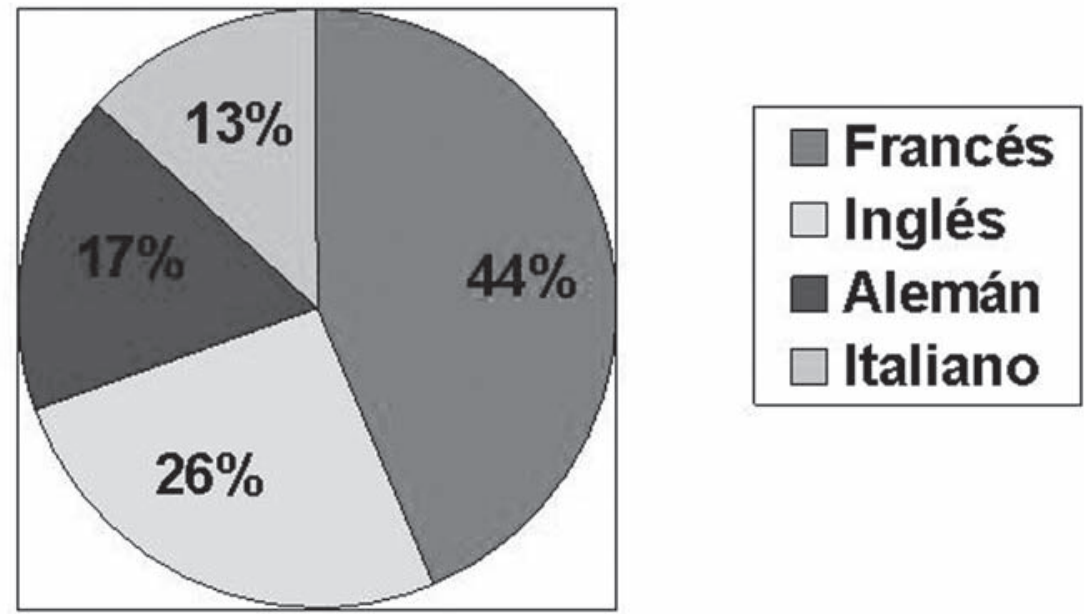

No es el objetivo de este artículo analizar los resultados obtenidos con el estudio bibliométrico con que se inicia esta investigación, así que nos limitaremos a reseñar cuál fue la metodología empleada de manera sistemática en ella. La información obtenida con este estudio, tal y como se intentó transmitir durante el seminario Work in Progress, nos ofrece una panorámica general de la situación de nuestro objeto de estudio y su manifestación en la historiografía, desde distintos puntos de vista, a un nivel cuantitativo, y con una perspectiva no solo actual, sino con una visión temporal, que nos indica la evolución que durante los años ha ido sufriendo la Asiriología en general, y la vida cotidiana de este pueblo en particular.

Pero si bien durante años se ha reivindicado el carácter cuantitativo que la Historia tiene, o debe tener, y que ha demostrado ser de gran relevancia, no hemos de dejar de lado la vertiente menos cuantificable, cualitativa, que una disciplina como la Historia debe tener en todo momento.

Por ello el estudio bibliométrico no es nuestro objeto de estudio, sino que comporta una parte más de él, ofrece información útil y que no siempre ha sido tenida en cuenta. Es más, en cierta medida nos sirve como punto de salida, como línea de inicio desde la que partir para el trabajo historiográfico. 
Una vez cuantificado la bibliografía y sacado las conclusiones que esto nos da, hemos de dar el siguiente paso, es decir, analizar esas obras, yendo más allá de los factores externos cuantificables.

\section{Metodología de la investigación}

Puesto que el objetivo del seminario Work in Progress es compartir con otros jóvenes investigadores la metodología empleada en nuestros trabajos, es este el apartado que más destacó durante la exposición oral de la presente investigación.

\section{Aspectos generales de la historiografía neoasiria}

La base del trabajo historiográfico llevado a cabo siguió la línea argumental de ir de lo general a lo concreto, alcanzando por tanto cada vez un mayor grado de concreción temática.

Se establecen así, en un primer momento, las características generales y más destacadas que se encuentran en la bibliografía, centrándonos en tres aspectos como vectores directores del análisis: autores, factores y argumentos.

Como muestra de la metodología desarrollada en este ámbito se destaca el trabajo concreto llevado a cabo con las diferentes problemáticas que se han detectado a lo largo de la investigación, destacado dos aspectos con notable preeminencia: en primer lugar los enfoques exógeneos con que con frecuencia se analiza históricamente al pueblo asirio, usando fuentes e incluso perspectivas propias de otros pueblos contemporáneos y/o vecinos del asirio.

En segundo lugar podemos destacar un tema recurrente en prácticamente la totalidad de las obras, la crueldad del pueblo asirio, que diversos autores sostienen o rechazan en mayor o menor grado, siendo esta última la tesis más extendida en las obras de más reciente publicación.

Siendo el asirio un pueblo tan frecuentemente denostado, la combinación de estos dos aspectos tan recurrentes ha generado una abundante historiografía en la que puede apreciarse, de forma más notoria o más soterrada, una predisposición negativa hacia su desarrollo y evolución, dada la enorme beligerancia asiria y su ineludible talante militar, lo que se percibe en prácticamente la totalidad de trabajos sobre los pueblos del Próximo Oriente Antiguo y el tratamiento para con el Imperio Asirio.

Llama la atención a este respecto los trabajos de algunos autores que consciente o inconscientemente soslayan la presencia asiria en época del Imperio Nuevo en sus publicaciones acerca de los diversos pueblos del Próximo Oriente en la primera mitad del primer milenio, siendo particularmente llamativo aquellos que nutren sus in- 
vestigaciones en buena medida de las fuentes neoasirias, ya sean estas arqueológicas como el palacio de Korshabad, o literarias, como la correspondencia real de época de Assurbanipal.

\section{La vida cotidiana en la historiografía}

El siguiente nivel de concreción al que hay que tener en cuenta es el tratamiento de aquellos aspectos directamente vinculados a la vida cotidiana en este periodo y que se recogen en el catálogo bibliográfico.

Lo primero que debemos plantearnos es qué entendemos por vida cotidiana. Bajo tan ambiguo epígrafe incluiremos aquellos temas a los que hacíamos referencia en la introducción del presente artículo, los que marcan el día a día de las gentes que habitan un territorio concreto en un momento histórico específico, con una concepción eminentemente amplia que incluye aspectos tan diversos como la alimentación, la diversión, la familia, la sexualidad, la educación o el vestido.

La historiografía del Próximo Oriente Antiguo en general, y del Imperio Asirio Nuevo como una muestra particular, presenta unas características específicas, que durante el seminario Work in Progress pudimos agrupar en cuatro:

- Es un objeto de estudio muy repartido, lo que se manifiesta en que para una investigación historiográfica que pretenda ser completa han de tenerse en cuenta un importante número de obras, pues, partiendo de la concepción amplia de la vida cotidiana anteriormente citada, la basta historiografía referida al ámbito neoasirio recoge en numerosas ocasiones datos concretos que puedan ser de utilidad para el objeto de nuestro estudio, pero su localización está repartida en múltiples trabajos.

- Siguiendo en esa misma línea, las obras dedicadas a tales temas con carácter específico son notablemente escasas, hasta el punto de que el repertorio bibliográfico ofrece casi un clamoroso silencio a primera vista, siendo necesario un estudio pormenorizado de la totalidad de las obras.

- De esas escasas obras que encontramos dedicadas como principal objetivo a la vida cotidiana en el Imperio Neoasirio, la inmensa mayoría la forman artículos de diversa extensión aunque nunca excesiva, con apenas algunas monografías especializadas.

- Cronológicamente, es obvio que mayoritariamente las obras que hacen referencia con mayor especificidad a la vida cotidiana se encuentran en el último tercio del siglo XX y los primeros años del XXI, aunque con ciertas excepciones, como se verá a continuación. 
Junto a estas características más destacadas, hay que citar una obra que supone una excepción para casi todas ellas, de ahí su importancia y relevancia, así como el especial análisis que de él se ha llevado a cabo en la presente investigación.

Nos referimos a la obra de Georges Contenau La vida cotidiana en Babilonia y Asiria, de $1951^{3}$ y que ofrece por primera vez un ejercicio histórico de recopilar todas las informaciones y datos que las diversas fuentes (literarias propias o externas, arqueológicas, etnológicas y artísticas) pueden mostrar de los múltiples aspectos que se aglutinan en la vida cotidiana del Imperio Asirio Nuevo.

Tal es la importancia de este pionero trabajo cuya estela siguen otros grandes especialistas como Bottéro ${ }^{4}$, Margueron ${ }^{5}$, Radner $^{6}$ o Albenda ${ }^{7}$ que en muchos de los aspectos relacionados con la vida cotidiana ha de tomarse ineludiblemente como punto de partida en la investigación historiográfica. Por ello se analiza con especial interés su estructura formal, el desarrollo de la obra y las conclusiones que aporta Contenau en ella.

A esto hay que sumarle el valor añadido de ser un magnífico ejemplo de la evolución historiográfica sufrida por los investigadores durante el siglo XX. De la Historia casi literaria, que hunde sus raíces en las obras decimonónicas de los pioneros en el Próximo Oriente Antiguo, se pasa a la Historia eminentemente científica, centrada en los datos empíricos. Y el tránsito entre un modo historiográfico y el otro se manifiesta en todo su esplendor en la obra de Contenau, con una nitidez infrecuente.

\section{La situación de la mujer en la historiografía}

Durante el seminario Work in Progress es difícilmente abarcable el plasmar todos y cada uno de los aspectos, temas y conclusiones tratados en una investigación doctoral, por lo que dado el formato del mismo seminario y la línea argumental de partir de lo general y alcanzar cada vez un mayor grado de concreción, se optó por plasmar únicamente uno de los aspectos de esa investigación, como muestra práctica y significativa de la metodología empleada y del trabajo desarrollado.

El tema elegido para ello fue el de la situación de la mujer de época neoasiria en la bibliografía anteriormente presentada y, por tanto, en la historiografía.

3 CONTENAU, G. La Vida cotidiana en Babilonia y Asiria. París. 1951 (Edición española de 1958, Barcelona).

4 BOTTÉRO, J. Annuaire de l'Ecole pratique des Hautes Etudes. Paris. 1966-67.

5 MARGUERON, J.C. Los Mesopotámicos. Paris. 1991. (Edición española de 1996, Madrid).

6 RADNER, K. Die neuassyrischen privatrechtsurkunden als Quelle für Mensch und Umwelt. Helsinki. 1997.

7 ALBENDA, P. The palace of Sargon, king of Assyria: monumental wall reliefs at Dur-Sharrukin, from original drawings made at the time of their discovery in 1843-1844 by Botta and Flandin. Paris. 1986. 
Tras analizar la bibliografía concreta para ello y recopilar las fuentes que sus autores emplean ${ }^{8}$, se ofrecen las características que los diferentes autores nos ofrecen, de este tema tan atrayente para la historiografía actual.

No cabe ninguna duda de que es este uno de los temas más trabajados por los especialistas en el Próximo Oriente Antiguo, desde distintas perspectivas, en especial la jurídica ${ }^{9}$ y sobre todo la ligada al matrimonio ${ }^{10}$. Dentro de estas perspectivas, es la de la vida cotidiana la que requiere nuestra atención.

En la presente investigación lo primero que se destaca por parte de los autores es la escasez de fuentes literarias específicas, que aporten información directa ${ }^{11}$, por lo que a menudo los únicos datos con los que se cuentan son los aportados de manera indirecta desde las fuentes mitológicas ${ }^{12}$ por extrapolación, artísticas ${ }^{13} \mathrm{o}$ arqueológi$\operatorname{cas}^{14}$.

Es frecuente encontrar en la historiografía la extrapolación, a veces excesiva pero siempre argumentada, de los casos concretos y notorios hacia la norma general. Muestra de ello es la llevada a cabo con los casos específicos de Semíramis, identificada por Roux como Sammuramat en sincretismo con la diosa Ishtar ${ }^{15}$ o de Naq'ia, en especial en el magnífico trabajo de Melville ${ }^{16}$ en los State Archives of Assyria Studies, cuya influencia en la corte neoasiria ha llevado a algunos autores a hablar del cierto feminismo en Senaquerib ${ }^{17}$.

Podemos resumir lo tratado durante el seminario Work in Progress en lo referente a este tema en que la mayor parte de los autores coinciden en que existe un endurecimiento de la situación de la mujer en el conjunto del Próximo Oriente Antiguo alrededor del año 1100 B.P. ${ }^{18}$ que le incapacita para ostentar el poder públicamente, limitando sus movimientos generalmente al ámbito doméstico y familiar (la materni-

8 Especial interés tiene para esta tarea el XXXIII Encuentro Internacional de Asiriología que bajo el título La Femme dans le Proche-Orient Antique fue celebrado en Paris en 1986.

9 RADNER, K. «Neoassyrian Period». A History of Ancient Near Eastern Law. Boston. 2003.

10 PELÁEZ DEL ROSAL, J. El divorcio en el derecho del Antiguo Oriente (Asiria, Babilonia, Israel). Córdoba. 1982.

11 FALES, F.M. «La estructura social». MOSCATI, S. (ed): El alba de la civilización. Sociedad, economía y pensamiento en el Próximo Oriente antiguo. Torino. 1976. (Edición Española de 1987, Madrid).

12 LAMBERT, W.G. «Goddesses in the Pantheon: a reflection of women in Society?». La Femme dans le Proche-Orient Antique. XXXIII Recontre Assyriologique Internationale. Paris. 1987

13 MARCUS, M.I. «Art and Ideology in Ancient Western Asia». CANE, 4: 2492

14 ALBENDA, P. op. cit, 1986.

15 ROUX, G. «Semíramis, la reina misteriosa de Oriente». Introducción al Antiguo Oriente. De Sumer a la Biblia. Paris. 1992. (Edición española de 1996, Barcelona).

16 MELVILLE, S.C. The role of Naqia/Zaqutu in Sargonid politics. Helsinki. 1999.

17 READE, J. «Was Sennacherib a Feminist?». La Femme dans le Proche-Orient Antique. XXXIII Recontre Assyriologique Internationale. Paris. 1987.

18 SAPORETTI, C. «The Status of the Women in the Middle Assyrian Period». MANE 2/1. 1979. 
dad), aunque con notables pero puntuales excepciones, achacándose todo ello a una sociedad patriarcal de origen semítico con ciertas influencias atenuantes sumerias ${ }^{19}$.

\section{Conclusión}

Una investigación historiográfica y bibliométrica debe ser capaz de aglutinar toda la información posible sobre el tema trabajado, y esa fue la intención de esta investigación y así se intentó transmitir a los asistentes a este seminario de jóvenes investigadores.

La metodología empleada se ha basado por tanto en tres principios:

1. Estudio de los datos cuantitativos de manera exhaustiva y detallada.

2. Estudio cualitativo de las investigaciones publicadas.

3. De lo general a lo concreto, estableciendo así distintos niveles de análisis.

El estudio histórico de la vida cotidiana en el Próximo Oriente Antiguo en general y en el Imperio Asirio Nuevo es una disciplina relativamente reciente, con un aumento cuantitativo de los trabajos, con notable predominio del formato en artículo y cuyo número de publicaciones va en progresivo aumento. Dentro de ellos hemos podido constatar el absoluto dominio de tres idiomas, inglés, alemán y francés.

Siendo conscientes de lo reciente de esta disciplina se pueden diferencias periodos con características propias. Si entre los años 1970 y 1990 nos encontramos ante una enorme diversificación temática que es muestra de una gran inquietud intelectual y de una profunda innovación historiográfica, a partir de 1990 presenciamos una mayor profundidad temática, síntoma de una mayor madurez de la disciplina, así como una mayor atención a la divulgación científica de los resultados de las investigaciones.

Pero esto no quiere decir que sea esta un área científica consagrada como tal. Siguen siendo escasos los trabajos especializados, pues si bien la Asiriología como ciencia goza de excelente salud, siguen predominando con absoluta entereza los enfoques políticos sobre los demás.

Por otro lado llama la atención que la mayoría de los autores observan un notable consenso en la mayor parte de los temas a debate, encontrándose escasas divergencias, y aquellas que sí existen no presentan una dificultad infranqueable.

Estas son algunas de las conclusiones que la presente investigación sobre la vida cotidiana del Imperio Neoasirio en la Historiografía nos han aportado y que fueron presentadas dentro del seminario Work in Progress a otros jóvenes investigadores,

19 BOTTÉRO, J. «Las libertades de las mujeres en Babilonia». Introducción al Antiguo Oriente. De Sumer a la Biblia. Paris. 1992. (Edición española de 1996, Barcelona). 
y que sientan la base para continuar el trabajo acerca de un pueblo tan fascinante y relevante como fue el Asirio.

\section{Bibliografía}

ALBENDA, P. «Woman, Child, and Family: their Imagery in Assyrian Art». La Femme dans le Proche-Orient Antique. XXXIII Recontre Assyriologique Internationale. Paris. 1987

ALBENDA, P. The palace of Sargon, king of Assyria: monumental wall reliefs at Dur-Sharrukin, from original drawings made at the time of their discovery in 1843-1844 by Botta and Flandin. Paris. 1986.

ASIMOV, I. El cercano Oriente. Boston. 1968. (Edición española de 2005, Madrid).

BEN-BARAK, Z. «The Queen consort and the struggle for succession to the throne».

La Femme dans le Proche-Orient Antique. XXXIII Recontre Assyriologique Internationale. Paris. 1987.

BOTTÉRO, J. «Las libertades de las mujeres en Babilonia». Introducción al Antiguo Oriente. De Sumer a la Biblia. Paris. 1992. (Edición española de 1996, Barcelona).

BOTTÉRO, J. La cocina más antigua del mundo. Paris. 2002. (Edición española de 2005, Barcelona).

BOTTÉRO, J. Annuaire de l'Ecole pratique des Hautes Etudes. Paris. 1966-67.

CAMINO GARCÍA, M. El cercano oriente: grandes imperios. Madrid. 1996.

CANCIK-KIRSCHBAUM, E. Die Assyrer: Geschichte, Gesellschaft, Kultur. München. 2003.

FALES, F.M. «La estructura social». MOSCATI, S. (ed): El alba de la civilización. Sociedad, economía y pensamiento en el Próximo Oriente antiguo. Torino. 1976. (Edición Española de 1987, Madrid).

FALES, F.M. L'impero assirio: stoira e amministrazione: (IX-VII secolo a.C.). Roma. 2001.

FINET, A. «El vino hace 5.000 años». Introducción al Oriente Antiguo. De Sumer a la Biblia. Paris. (Edición española de 1996, Barcelona). 1992.

FUCHS, A. Die Annalen des Jahres 711 v. Chr. nach Prismenfragmenten aus Ninive und Assur. Helsinki. 1998.

GONZÁLEZ TORRES, A.L. «La familia neoasiria en la historiografía moderna». PANTA REI, 2, II época. Murcia.2007.

GRAYSON, A.K. Assyrian rulers of the early first millennium BC I (1114-859 BC). Toronto. 1991.

HOLLOWAY, S.W. Assur is king! Assur is king! Religion in the exercise of power in the Neo-Assyrian Empire. Boston. 2002. 
HROUDA, B. Die Assyrer und ihre Kunst in neur Sicht. München. 2003.

JAS, R. Neo-Assyrian judicial procedures. Helsinki. 1996.

KLÍMA, J. Sociedad y cultura en la Antigua Mesopotamia. Praga. 1964. (Edición española revisada de 1995, Madrid).

KRAMER, N.S. La historia empieza en Sumer. New York. 1959. (Edición española de 1985, Barcelona).

KUHRT, A. El oriente en la antigüedad, I1. C. 3000-330 A. C. London. 1995. (Edición española de 2001, Barcelona).

LAFONT, B. «Las mujeres del palacio de Mari». Introducción al Antiguo Oriente. De Sumer a la Biblia. Paris. 1992. (Edición española de 1996, Barcelona).

LAMBERT, W.G. «Goddesses in the Pantheon: a reflection of women in Society?». La Femme dans le Proche-Orient Antique. XXXIII Recontre Assyriologique Internationale. Paris. 1987.

LIVERANI, M. El Antiguo Oriente. Historia, sociedad, economía. Roma. 1988. (Edición española de 1995, Barcelona).

MARCUS, M.I. «Art and Ideology in Ancient Western Asia». CANE, 4: 2492

MARGUERON, J.C. Los Mesopotámicos. Paris. 1991. (Edición española de 1996, Madrid).

MELVILLE, S.C. The role of Naqia/Zaqutu in Sargonid politics. Helsinki. 1999.

NIESSEN, H.J. Von Mesopotamien zum Irak: Kleine Geschcichte eines alten Landes. Berlin. 2004.

PARPOLA, S and PORTER, M. The Helsinki atlas of the Nea East in the neo-assyrian period. Helsinki. 2001.

PELÁEZ DEL ROSAL, J. El divorcio en el derecho del Antiguo Oriente (Asiria, Babilonia, Israel). Córdoba. 1982.

RADNER, K. «Neoassyrian Period». A History of Ancient Near Eastern Law. Boston. 2003.

RADNER, K. Die neuassyrischen privatrechtsurkunden als Quelle für Mensch und Umwelt. Helsinki. 1997.

RADNER, K. Die neuassyrischen Texte aus Tall Seh Hamad. Berlin. 2002.

READE, J. «Was Sennacherib a Feminist?». La Femme dans le Proche-Orient Antique. XXXIII Recontre Assyriologique Internationale. Paris. 1987

ROUX, G. «Semíramis, la reina misteriosa de Oriente». Introducción al Antiguo Oriente. De Sumer a la Biblia. Paris. 1992. (Edición española de 1996, Barcelona).

ROUX, G. Mesopotamia. Historia política, económica y cultural. Paris. 1985. (Edición española de 2002, Madrid).

SANMARTÍN, J. Códigos legales de tradición babilónica. Madrid. 1999. 
SANMARTÍN, J. y SERRANO, J.M. Historia antigua del Próximo Oriente. Mesopotamia y Egipto. Madrid. 1998.

SAPORETTI, C. «The Status of the Women in the Middle Assyrian Period». MANE 2/1. 1979.

SODEN, W.v. Introducción al orientalismo antiguo. Darmstadt. 1985. (Edición española de 1987, Barcelona).

WALKER, J.M. Las civilizaciones del Próximo Oriente. Madrid. 1996 